\title{
Large reservoir subcutaneous pump implantation
}

\section{SRT surgical narrative}

The goal of this survival surgical procedure is to successfully implant a large reservoir subcutaneous pump in a mouse. All surgeries are conducted in a surgical suite specifically designated for rodent surgery. The surgeon wears clean scrubs, shoes designated only for use in the vivarium, a disposable paper gown, a hair bonnet, a face mask, and sterile gloves. The mouse is obtained from an approved vendor and has met the Institute's 72hour acclimation requirement prior to surgery or was bred on site. For the purpose of this narrative, a 15 week old male mouse is assumed. First, the surgical area is prepared by placing out all instruments and suture necessary for the procedure including the pre-filled subcutaneous pumps for implantation, scissors, blunted scissors, and suture on to a sterile drape. This creates the sterile field. These items have been sterilized by autoclaved or purchased pre-sterilized.

Before anesthetizing the mouse, he is injected with an analgesic $(0.05 \mathrm{mg} / \mathrm{kg}$ buprenorphine, subcutaneously) for pre-operative pain management as required by the IACUC. The mouse is placed in an induction chamber in which the flow rate of isoflurane anesthesia is set to between 2 and 3 percent. The oxygen remains at $1 \mathrm{~L} / \mathrm{min}$ while the percentage of inhaled isoflurane is adjusted accordingly throughout the procedure. To ensure the animal is in the correct surgical plane of anesthesia, constant attention is paid to the animal's respiratory pattern and response to stimuli. The animal should not appear overly sedated with heavy or labored breathing. Alternately, he should not have shallow or rapid breathing. A toe pinch test is performed on the mouse by pinching a hind foot. There should be no movement in response to the toe pinch. Once the mouse is in the correct surgical plane of anesthesia, he is taken to a pre-operative preparation area in the surgical suite and his interscapular region is promptly shaved. The mouse is returned back to the isoflurane anesthesia induction chamber to ensure that he is in the correct surgical plane of anesthesia. Next, the mouse is placed in the surgical field in a prone position. In addition to the laid out instruments and supplies, this area consists of a stainless steel plate that sits on top of a warming surface to maintain the mouse's body temperature during the procedure and. The surgical area also consists of a stereomicroscope. Sterile ophthalmic ointment is applied onto both eyes of the mouse to prevent drying during the surgery. His head is secured into a nose cone that is connected to the isoflurane anesthesia machine. To perform aseptic preparation of the surgical incision area, a cotton swab is used to scrub the shaved interscapular area in a ciricular motion with Betadine (povidoneiodine) scrub followed by $70 \%$ isopropyl alcohol. This is repeated and finished with Betadine solution which is left to dry. Next, the animal is covered with sterile drape, revealing his prepared shaven area.

With a pair of forceps, the shaved area of skin is lifted and scissors are used to make a $5 \mathrm{~mm}$ incision in between his shoulder blades. Blunt tipped scissors are inserted into the incision site and proceeded along down the length of the animal's spine. The scissors are slowly opened wide while they are positioned underneath the skin of the mouse to delicately tease away at any connective tissue which will detach the skin from the muscle. Once the scissors are opened and closed three or four times and tension is no longer felt, the blunted scissors are removed. This method creates a subcutaneous pocket for
Volume 4 Issue 2 - 2016

\author{
Emily King BS \\ Sanford-Burnham Medical Research Institute, USA
}

Correspondence: Emily King, BS, Research Associate I, Sanford-Burnham Medical Research Institute at Lake Nona, Cardiometabolic Phenotyping Core, 6400 Sanger Road, Orlando, FL 32827, USA, Tel (407) 745 2000, Extn 6007, Email eking@sanfordburnham.org

Received: July 08, 2016 | Published: November 10, 2016

the pump to sit and also allows for unobstructed flow of the pump's infusate. Next, the pump is inserted into the incision site so that the delivery portal is facing towards the hind limbs of the animal. It is gently slid down the spine of the mouse near the base of his hind limbs using the thumb and forefinger. To verify a proper pocket is created, the pump should slide easily into position. There should be neither resistance nor tension when inserting the pump into the pocket. The incision is sutured closed using 6-0 silicon coated braided silk suture with a $3 / 8$ inch $12 \mathrm{~mm} \mathrm{C}-1$ cutting edge needle in an uninterrupted suture pattern.

Finally, the isoflurane and oxygen are turned off. $0.05 \mathrm{mg} /$ $\mathrm{kg}$ buprenorphine analgesic is administered subcutaneously postoperatively per the IACUC protocol. His body weight is recorded along with any remarkable observations on his surgery cage card. The mouse in placed in lateral recumbency in a warmed cage with the bedding removed. In approximately 2 minutes, once the animal is ambulatory, the mouse is then singly housed on a thermal barrier in the vivarium. The large reservoir subcutaneous pump implantation surgical procedure is a survival surgery in which the animal is expected to live a normal lifespan. Therefore, constant clinical monitoring is imperative to ensure the health of the animal while the pump is in place. The animal is weighed daily and allowed to lose no more than $20 \%$ of his body weight for consideration as a candidate for continued experimentation. Additionally, the mouse is checked daily for signs of pain, distress, and suffering by noting any changes in behavior and appearance. The mouse should appear active and curious. Observances are made for lethargy, hunched body posture, a rough unkempt fur coat, and signs of dehydration. A good indicator of a dehydrated mouse is done by performing a tent test in which the skin over his shoulder blades is picked up with the forefinger and thumb. It should return to normal position quickly and not stay in a tented or bunched position. Another indication of dehydration is the appearance of recessed or sunken-in eyes. Occasionally self-mutilation can occur. The incision sites are observed for redness and inflammation or any weeping and oozing which can indicate a sign of infection. If the animal is found to exhibit the indicators mentioned above he is recommended for termination from the study and possibly early euthanasia. The attending Veterinary Technician on site is notified of these observations as well as the responsible party from the laboratory that is conducting the research study. In addition to the surgeon's monitoring, the animal is identified as a surgery animal and monitored by the vivarium staff for on weekends and holidays. 


\section{Acknowledgements}

None.

\section{Conflict of interest}

Author declares that there is no conflict of interest. 